\title{
AN EXTENSION OF TURÁN'S INEQUALITY
}

\section{Geno Nikolov and Veronika Pillwein}

Abstract. Let $p_{m}(x)=P_{m}^{(\lambda)}(x) / P_{m}^{(\lambda)}(1)$ be the $m$-th ultraspherical polynomial normalized by $p_{m}(1)=1$. We prove the inequality $|x| p_{n}^{2}(x)-p_{n-1}(x) p_{n+1}(x) \geqslant 0, x \in[-1,1]$, for $-1 / 2<$ $\lambda \leqslant 1 / 2$. Equality holds only for $x= \pm 1$ and, if $n$ is even, for $x=0$. Further partial results on an extension of this inequality to normalized Jacobi polynomials are given.

Mathematics subject classification (2010): 41A17, 68W30.

Keywords and phrases: Turán inequality, orthogonal polynomials, ultraspherical polynomials, symbolic computation, cylindrical algebraic decomposition.

\section{REFERENCES}

[1] H. Alzer, S. Gerhold, M. Kauers, And A. Lupaş, On Turán's inequality for Legendre polynomials, Expo. Math., 25(2): 181-186, 2007.

[2] C. Berg And R. Szwarc, Bounds on Turán determinants, J. Approx. Theory, 161 (1): 127-141, 2009.

[3] C. W. BROWN, QEPCAD B - a program for computing with semi-algebraic sets Sigsam Bulletin, 37 (4): 97-108, 2003.

[4] C. Chen, M. MazA, M. B. XIA, AND L. YANG, Computing cylindrical algebraic decomposition via triangular decomposition, In ISSAC 2009-Proceedings of the 2009 International Symposium on Symbolic and Algebraic Computation, pages 95-102. ACM, New York, 2009.

[5] G. E. Collins, Quantifier elimination for real closed fields by cylindrical algebraic decomposition, In Automata theory and formal languages (Second GI Conf., Kaiserslautern, 1975), pages 134-183. Lecture Notes in Comput. Sci., Vol. 33. Springer, Berlin, 1975.

[6] D. K. Dimitrov And G. P. Nikolov, Sharp bounds for the extreme zeros of classical orthogonal polynomials, J. Approx. Theory, 162 (10): 1793-1804, 2010.

[7] A. Dolzmann AND T. STURM, Redlog: computer algebra meets computer logic, Sigsam Bulletin, 31 (2): 2-9, 1997.

[8] G. GASPer, On the extension of Turán's inequality to Jacobi polynomials, Duke Math. J., 38: 415428, 1971.

[9] G. Gasper, An inequality of Turán type for Jacobi polynomials, Proc. Amer. Math. Soc., 32: 435439, 1972.

[10] S. Gerhold And M. Kauers, A Procedure for Proving Special Function Inequalities Involving a Discrete Parameter, In Proceedings of ISSAC '05, pages 156-162. ACM Press, 2005.

[11] S. Gerhold AND M. KAUERS, A computer proof of Turán's inequality, Journal of Inequalities in Pure and Applied Mathematics, 7 (2): \#42, 2006.

[12] M. Kauers, SumCracker - A Package for Manipulating Symbolic Sums and Related Objects, Journal of Symbolic Computation, 41 (9): 1039-1057, 2006.

[13] M. KAUERS, Computer algebra and power series with positive coefficients, In Proceedings of FPSAC'07, 2007.

[14] M. Kauers, Computer algebra and special function inequalities, In Tewodros Amdeberhan and Victor H. Moll, editors, Tapas in Experimental Mathematics, volume 457 of Contemporary Mathematics, pages 215-235. AMS, 2008.

[15] M. Kauers, How To Use Cylindrical Algebraic Decomposition, Seminaire Lotharingien de Combinatoire, 65 (B65a): 1-16, 2011. 
[16] M. KAUers AND P. PAUle, A computer proof of Moll's log-concavity conjecture, Proceedings of the AMS, 135 (12): 3847-3856, 2007.

[17] G. NiKOLOv, Inequalities of Duffn-Schaeffer type, II, East J. Approx., 11 (2): 147-168, 2005.

[18] V. PILlwein, Positivity of certain sums over Jacobi kernel polynomials, Advances in Applied Mathematics, 41 (3): 365-377, 2008.

[19] A. SEIDL AND T. STURM, A generic projection operator for partial cylindrical algebraic decomposition, In Proceedings of the 2003 International Symposium on Symbolic and Algebraic Computation, pages 240-247 (electronic), New York, 2003. ACM.

[20] A. StrZeboński, Solving systems of strict polynomial inequalities, J. Symbolic Comput., 29 (3): 471-480, 2000.

[21] Oтto SZÁsz, Identities and inequalities concerning orthogonal polynomials and Bessel functions, J. Analyse Math., 1: 116-134, 1951.

[22] G. SZEGő, On an inequality of P. Turán concerning Legendre polynomials, Bull. Amer. Math. Soc., 54: 401-405, 1948.

[23] G. Szegő, Orthogonal polynomials, American Mathematical Society Colloquium Publications, Vol. 23. Revised ed. American Mathematical Society, Providence, R.I., 1959.

[24] RysZard SZWARC, Positivity of Turán determinants for orthogonal polynomials, In Harmonic analysis and hypergroups (Delhi, 1995), Trends Math., pages 165-182. Birkhäuser Boston, Boston, MA, 1998.

[25] V. R. ThiruvenKatachar And T. S. NANJUndiah, Inequalities concerning Bessel functions and orthogonal polynomials, Proc. Indian Acad. Sci., Sect. A., 33: 373-384, 1951. 